\title{
Rue et marginalité : le cas de Londres au XIX siècle
}

The Street and the Margin: the Case of $19^{\text {th }}$ Century London

\section{Didier Revest}

\section{(2) OpenEdition}

1 Journals

\section{Édition électronique}

URL : http://journals.openedition.org/rfcb/1599

DOI : 10.4000/rfcb.1599

ISSN : 2429-4373

Éditeur

CRECIB - Centre de recherche et d'études en civilisation britannique

\section{Édition imprimée}

Date de publication : 1 septembre 2003

ISSN : 0248-9015

\section{Référence électronique}

Didier Revest, "Rue et marginalité : le cas de Londres au XIXe siècle », Revue Française de Civilisation Britannique [En ligne], XII-3 | 2003, mis en ligne le 01 septembre 2003, consulté le 19 avril 2019. URL: http://journals.openedition.org/rfcb/1599; DOI : 10.4000/rfcb.1599

Ce document a été généré automatiquement le 19 avril 2019

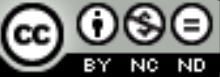

Revue française de civilisation britannique est mis à disposition selon les termes de la licence Creative Commons Attribution - Pas d'Utilisation Commerciale - Pas de Modification 4.0 International. 


\title{
Rue et marginalité : le cas de Londres au XIX ${ }^{\mathrm{e}}$ siècle
}

\author{
The Street and the Margin: the Case of $19^{\text {th }}$ Century London
}

Didier Revest

'When Idleness is removed, Poverty is removed
likewise (...).'
Josiah Tucker, The Elements of Commerce and the
Theory of Taxes, 1755

Quelle que soit la société étudiée, il n'existe guère que deux types de marginalité : celle que l'on choisit - on est alors un(e) marginal(e) - et celle qui est subie - on est alors marginalisé(e $)^{1}$. Le premier cas de figure est, de notre point de vue, peu intéressant, car il est à sens unique et n'implique que le libre-arbitre d'une seule personne ou d'un groupe très minoritaire. Le second, en revanche, l'est beaucoup plus. En effet, ici, la notion de marge présuppose celle de centre. En d'autres termes, il n'y a de(s) marge(s) qu'en fonction d'un centre qui en définit nature et contours. La marginalité pour l'essentiel n'est donc pas, pour reprendre à notre compte une expression de Jacques Le Goff, " un fourre-tout rempli par un bric-à-brac »; il y a bien plutôt existence d'un processus qui conduit à la marginalisation, celle d'une frontière tracée par la société entre marginalité et normalité, fondée sur des justifications idéologiques de l'exclusion ${ }^{2}$. Partant, les marges ne sont pas des réalités absolues, objectives, mais bel et bien le fruit d'un arbitraire discursif, lui-même fruit d'une époque, d'une culture :

(La marginalité) joue comme une fonction-miroir de la société. En effet, pour chaque prise de position la concernant, elle révèle la société car elle lui permet de se saisir dans le rapport qu'elle entretient avec ses exclus ${ }^{3}$.

2 S'intéresser aux marges de la ville victorienne, à commencer par Londres, cela revient donc pour une large part, essentielle, à analyser ce qui les faisait naître, à savoir la morale et le discours dominants, ceux des classes aisées. C'est pourquoi nous analyserons en tout premier lieu les catégories sociales les plus mobiles, c'est-à-dire les plus susceptibles d'entrer dans cette marginalité nommée d'en haut que nous avons essayé de définir, c'est-à-dire les prostituées, les mendiants et vagabonds, la populace, bref, les pauvres, 
qui, plus ouverts que les autres groupes de la société aux idées égalitaires, sont tenus par les classes dirigeantes pour de possibles agents de subversion sociale et politique, mais aussi les délinquants, perçus la plupart du temps comme n'ayant ni nom, ni visage. Puis, nous dirons en quoi ces groupes étaient loin d'être marginaux, avant de souligner ce qui, à notre avis, a contribué à faire naître et prospérer ce contresens tenace. Nous verrons pour finir comment le débat sur la pauvreté s'en est trouvé totalement figé, pour le plus grand malheur de tout un pan de la population londonienne.

\section{Marginalité ou misère?}

3 À en croire les sources primaires, le plus vieux métier du monde semble avoir été dans les années 1830 et 1840 déjà un des traits caractéristiques des rues de Londres. On dénonçait alors la présence envahissante des prostituées, peut-être 17000 en tout ${ }^{4}$, notamment dans le West End. Les archives pour les années postérieures à 1860 regorgent de lettres et pétitions contre la prostitution, signées par des particuliers constitués en associations, par des conseils curiaux, etc. ${ }^{5}$. Vers 1900 encore, les rues du West End (Piccadilly Circus, les rues aux abords de la gare Victoria, etc.) demeuraient les hauts lieux de la prostitution ${ }^{6}$. Certes, il était des rues qui semblaient ne plus l'attirer, mais cela cachait une double mutation de la prostitution. Si la zone de Charing Cross et du Strand était moins fréquentée en 1900 qu'en 1880, c'était en raison du déplacement de la prostitution vers Mayfair et Bayswater entre autres ${ }^{7}$, sans parler de toutes ces femmes qui, entre-temps, étaient allées grossir les rangs des prostituées déjà nombreuses de l'East End.

4 Les mendiants, de leur côté, auront été, dès les années 1830 , perçus comme un groupe en constante augmentation. De 8000 à cette époque, leur nombre serait passé à quelque 15 000 vers $1860^{8}$. Cette augmentation semble être allée de pair avec celle du nombre d'errants dans l'absolu, dont le nombre passe de 20000 environ en 1835 à 70000 à la fin des années $1850^{\circ}$. Ils ne connaissaient, pour ainsi dire, que la rue; ayant pour tout lit une embrasure de porte ou une arche de pont de chemin de fer, ils souffraient des maladies qui affectent en priorité ceux qui n'ont plus rien : bronchite, rhumatismes, etc. ${ }^{10}$.

5 Vers 1860 en outre, il y aurait eu à Londres 16000 'street Arabs' (enfants sans domicile fixe) des deux sexes, ${ }^{11}$ forcés de battre le pavé pour les raisons suivantes : décès d'un père et/ou d'une mère, alcoolisme d'un père adoptif, difficultés dues à la faillite du commerce paternel, mais aussi brutalités bien souvent. Autant d'éléments qui amenèrent le président du London School Board à déclarer en 1875 :

The thousand of fusee vendors, newspaper boys, street-sweepers, and what not, if within their age, are individually known and their school attendance closely watched. But, beyond these, we have a crowd of half-famished, half-naked children who prowl about alleys and railway-arches, fruit markets and the river foreshore, the difficulty of pressing whom into school is almost insuperable. They are no man's children, and live on no man's land (... $)^{12}$.

6 Les choses ne semblent guère s'être améliorées par la suite. Dans une lettre type de décembre 1887, dont il envoyait alors de nombreux exemplaires pour obtenir des fonds en prévision de l'hiver qui s'annonçait ${ }^{13}$, Barnardo déclarait :

More homeless children are now to be seen wandering about, or asleep at night in our streets, than ever before. Each day crowds of starving little ones besiege our gates $»^{14}$. 
7 Toutes les formes de délinquance paraissaient elles aussi faire tache d'huile. Dans New Cut (Lambeth) par exemple, durant la décennie 1840, de vieilles marchandes ambulantes voyaient fréquemment, le soir, leurs étals pris d'assaut par des bandes de garnements qui, non contents de les bousculer, leur dérobaient ce que bon leur semblait avant de s'enfuir ${ }^{15}$ . La police pour sa part était impuissante à empêcher les pauvresses des rues les plus insalubres du Borough ou de l'East End de venir chaparder aux étalages des magasins de Lambeth Walk ou de Tottenham Court Road ${ }^{16}$, ou à prévenir cette forme de délinquance qui consistait à détrousser les personnes ivres ${ }^{17}$, comme c'était le cas dans Westminster Road, le Strand, Thames Street, et bien d'autres.

8 Les années 1850 et le début de la décennie suivante virent même une nouvelle forme de délinquance, la Garotte, prospérer sur le pavé londonien. L'Annual Register de 1862 définissait ainsi cette pratique : tandis que l'un des deux assaillants (quelquefois trois) entourait le cou d'un passant de son bras afin de lui comprimer la gorge, voire de l'assommer, son acolyte lui faisait les poches ${ }^{18}$. George Godwin, rédacteur en chef de la revue The Builder, indique d'ailleurs que, durant ces années-là, la capitale pullulait de malfrats et voleurs en tout genre, 250000 en tout selon lui ${ }^{19}$.

Dans l'East End enfin, les agressions contre la police étaient légion (dix cas furent ainsi rapportés pour les six premiers mois de l'année 1888 par l'East End Observer) ${ }^{20}$; on s'y attaquait également aux passants, aux marins (pratique appelée 'poncing'21), aux enfants ( 'kynchin lay'22), aux juifs, aux marchands ambulants, auxquels on dérobait montres, argent, etc. Au plus fort de la série meurtrière de Jack L'Éventreur, il se trouvera même un député (M. Pickersgill, élu de Derby) pour rappeler, lors de son intervention aux Communes le 14 novembre 1888, que la question la plus grave n'était pas celle des meurtres commis par le «monstre de l'East End", mais bel et bien '(...) that disorderly condition of the public streets which [has] grown up under the late administration (...)'.

10 À ce stade, il est important de se demander si prostituées, mendiants, vagabonds et délinquants étaient tous des marginaux, s'ils s'étaient tous exclus d'eux-mêmes. Comme nous allons le voir, c'est par la négative qu'il faut répondre. La situation de Londres au $\mathrm{XIX}^{\mathrm{e}}$ siècle vient tout simplement confirmer la théorie, applicable à d'autres villes et époques, selon laquelle la marginalité perçue est un des corollaires immédiats de la pauvreté.

11 Chez les prostituées, considérées comme l'archétype de la déviance, on a certes bien une frange de vagabondes sans logis, dont beaucoup n'ont pas vingt ans, et que la prostitution nourrit, au même titre que la mendicité, depuis l'âge de dix ou douze ans ${ }^{23}$. Mais la majorité font commerce de leur corps dans des lupanars situés au sein de quartiers et de communautés où elles sont connues. La plupart sont de surcroît intégrées à la société puisqu'elles ont un métier qu'elles exercent le jour, mais un métier trop mal rémunéré pour survivre, d'où la nécessité de se prostituer. Impossible d'assurer autrement ses fins de mois, comme l'ont expliqué ces deux ouvrières du textile :

$1^{\text {ère }}$ ouvrière : I do the best I can with what little I earn, and the rest I am obligated to go to the streets for...I can't get a rag to wear without flying to prostitution for it. $2^{e}$ ouvrière: (...) I was forced to depend entirely upon the streets for my food. On my soul, I went to the streets solely to get a living for myself and my child. ${ }^{24}$

12 Ainsi que le déclarait encore une prostituée dans une longue lettre intitulée 'The Great Social Evil' et publiée dans le Times du 24 février 1858 : 
It is a cruel calumny to call them in mass prostitutes (...), these poor women toiling on starvation wages, while penury, misery, and famine clutch them by the throat and say, 'Render up your body or die'25. traditions et rapports de force qui liaient ces enfants/adolescents, n'étaient pas le sceau de la marginalité, mais plutôt la preuve qu'ils s'étaient structurés afin, tout bonnement, de survivre. Car les membres de cette catégorie, comme ceux des autres groupes, qui sont pour le profane autant de mystères difficiles à percer, vivaient en réalité des existences qui étaient un puissant facteur d'union ${ }^{31}$.

17 S'agissant de la délinquance, il serait absurde de la minimiser, car elle faisait partie intégrante de la vie dans les rues de Londres. Néanmoins, pas plus que les autres, les délinquants, sûrement moins nombreux qu'on a bien voulu le dire, ne vivaient en marge de la société. Il suffisait de se rendre dans Lower Keat Street (Whitechapel), dans Gunn Street et Market Street (Borough), etc., pour voir, de jour comme de nuit, les vrais « seigneurs » des lieux, voleurs et souteneurs, généralement bien mis, adossés au mur de quelque pub, ou devisant innocemment avec leurs semblables sur le trottoir ou au milieu de la chaussée ${ }^{32}$. Les réseaux de receleurs avaient même pignon sur rue puisqu'ils faisaient partie d'une économie certes parallèle et illégale, mais parfaitement structurée et située au cœur de la société londonienne. Dans Field Lane, proche de Holborn Hall, on vendait en toute impunité des mouchoirs d'occasion dérobés quelques heures auparavant par de petites mains fort habiles ; ce commerce parfaitement illicite, qui attirait de nombreuses personnes, la nuit notamment, était pourtant pratiqué le plus ouvertement du monde ${ }^{33}$. La plupart des délinquants semblent en outre avoir été connus des services de police au moins, ainsi ceux qui sévissaient lors des marchés qui se tenaient dans Petticoat Lane durant les années 1880 par exemple ${ }^{34}$. 
18 Autre marginalité perçue, la populace, dont les rassemblements faisaient resurgir de vieilles peurs, et particulièrement celle de la révolution. Lord Liverpool n'avait-il pas déclaré à Chateaubriand en 1810 déjà, qu'il suffisait d'une seule insurrection à Londres pour que tout soit perdu ${ }^{35}$ ? Mais comme le pensaient certains, dont Karl Marx, ces phénomènes que l'on disait à caractère révolutionnaire, étaient d'abord le fait d'une masse indistincte d'êtres humains qui se liguaient spontanément, sans concertation préalable, et qui, le reste du temps, étaient totalement "invisibles». Les émeutes de Whitechapel et Mile End du 16 janvier 1861 entre autres n'avaient eu lieu qu'en raison d'un hiver particulièrement rude et des difficultés éprouvées par des milliers de dockers poussés à bout ${ }^{36}$.

Certes, John Burns, le 8 février 1886, lors des manifestations de Trafalgar Square à l'occasion du rassemblement du London Working Men's Committee suscité par le chômage et la misère, avait bien - muni d'un drapeau rouge - harangué la foule en prônant la révolution, tout en se référant aux Canuts lyonnais de 1834 ainsi qu'aux Chartistes ${ }^{37}$. Quant aux journées du 9 et du 10 février 1886, elles avaient porté à son paroxysme la peur de la rébellion généralisée. En divers lieux (Whitechapel, Camden Town, Southwark), la rumeur selon laquelle des bandes de voyous s'apprêtaient à fondre sur le West End et la Cité s'était répandue. On avait même parlé de 30000 émeutiers marchant sur Trafalgar Square. Pourtant, hormis quelques échauffourées entre la police et quelques centaines de mauvais garçons, l'hydre révolutionnaire épargnera la capitale ${ }^{38}$.

On n'a en effet retenu des aspirations légitimes du pauvre que les noms de certains meneurs et les exactions auxquelles une petite minorité se livrait. On n'a donc eu d'yeux que pour l'aspect le plus inquiétant et le plus laid des manifestations. Ce faisant, le pouvoir et les classes aisées ont envisagé la foule comme un bloc monolithique, ne songeant pas un instant à tenter d'en déchiffrer les "attitudes plurielles ${ }^{39}$. Loin de ne condamner que ce qui était pénalement condamnable (attaques de boutiques, bris de vitres, ou bataille rangée avec les forces de l'ordre), on a voué aux gémonies l'intégralité des agissements de ces hommes et femmes, qui ne demandaient qu'à survivre.

21 Une nouvelle question mérite maintenant d'être posée : comment les classes aisées en sont-elles arrivées à voir ici, comme partout ailleurs, de la marginalité?

\section{Une affaire de regard}

22 Se méfier du vagabond, à commencer par lui, le montrer du doigt en raison de ses turpitudes morales supposées, était pour ainsi dire inscrit dans les mœurs. Depuis le vote du statut de 1349 contre, entre autres, les faux mendiants, le pays avait connu de nombreuses vagues de répression à l'encontre de ceux que l'on percevait comme des marginaux : campagne nationale entre 1569 et 1572 contre les errants; puis naissance de la maison de correction; et enfin vote des lois de 1598 et 1600 sur le vagabondage, qui fixèrent pour près de trois siècles les modalités de répression du phénomène ${ }^{40}$.

23 A vrai dire, les élites en vinrent à se méfier de bien des catégories sociales. Comme l'a rappelé Michel Foucault, à la suite de Pierre Chaunu, la multiplication des propriétés et des richesses, à partir de la seconde moitié du XVIII siècle, avait fait naître, chez les possédants, un "besoin de sécurité »". Au début du siècle suivant, cette affirmation de la propriété alla de pair avec une autre conséquence du capitalisme, à savoir l'essor d'une classe ouvrière plus nombreuse que jamais, dans une ville immense où elle donnait dès 
lors l'impression d'échapper à tout contrôle, d'habiter un univers décalé, aux marges floues de la nouvelle société, elle-même fondée désormais sur l'opposition des classes sociales ${ }^{42}$.

C'était l'incertitude de leur état qui éveillait soupçon et peur. C'était la mobilité non cataloguée à l'intérieur de la capitale, laquelle empêchait beaucoup d'hommes et de femmes d'avoir un statut bien défini selon les canons de l'époque, qui les rejetait immanquablement hors des cadres sociaux établis ${ }^{43}$. L'évolution, lente mais sûre, de la société favorisait de plus la mise en marge d'une partie de la population. Car «plus la société occidentale est entrainée dans la rationalisation et l'éclaircissement de ses normes [grâce, dans le cas de Londres, à une surveillance qui se généralise: naissance de la police métropolitaine en 1829, et de celle de la Cité en 1839 par exemple], plus elle assombrit ses perceptions de l'anormalité, et plus elle devient sensible à la simple différence et à la déviance $"{ }^{44}$.

La presse a joué un rôle capital dans ce processus; car, étant le lien principal entre une certaine société, d'un côté, et la rue et son peuple, de l'autre, et dénonçant tout ce qui n'était pas - à ses yeux - clairement visible, c'est essentiellement elle qui a inventé le monde effrayant et obscur de la délinquance de rue à grande échelle. L'émoi, en effet, qui saisit Londres au moment de l'affaire de la Garotte (à partir de juillet 1862 surtout) doit toute sa réalité non pas tant à des délits graves commis sur la voie publique, mais plutôt à l'interaction entre la presse et ses lecteurs : prompte à l'amalgame et partisane, elle nourrissait les angoisses de son lectorat qui, en retour, noircissait de ses lettres, et de ses expériences malheureuses éventuelles, les colonnes des journaux. Que la teneur des lettres envoyées au Times par exemple ait fait écho à celle des articles parus dans ce même quotidien ne fait aucun doute. Lorsque la violence décrite par ses journalistes augmentait d'un cran, les lettres reçues devenaient plus radicales ${ }^{45}$. Le 23 novembre 1862, The Observer, comme The Spectator précédemment (le 19 juillet 1862), déplorait ainsi que les rues ne fussent plus ce qu'elles avaient étée ${ }^{46}$. Dans la journée, elles semblaient être devenues aussi inquiétantes qu'en période nocturne. Le Daily News du 18 juillet 1862 n'hésita pas à écrire que les rues étaient le « champ de bataille de cochers de fiacre en furie le jour, et un repaire de détrousseurs et d'assassins la nuit $»^{47}$. Il n'est pas étonnant alors qu'on ait pu lire le 5 novembre 1862 par exemple sous la plume d'un lecteur que "les nuits devenant plus longues, il nous faudrait acheter des revolvers et les transporter dans nos poches $»^{48}$.

Nous savons en réalité aujourd'hui que la phase de panique a bel et bien précédé le décollage du nombre de cas d'agression recensés (l'effet a donc précédé la cause !). A cela une raison simple : la police, qui avait l'habitude de subir de plein fouet les critiques des journaux l'accusant de laxisme, a fait preuve d'un zèle accru, car elle-même a souscrit pour un temps au moins - à l'existence d'une lame de fond, comme le prouvent les ordres transmis le 18 juillet 1862 :

Robberies in the streets - in reference to cases of robbery in the streets that have recently been committed, the police on beats at night are to make special observation on dark passages, entries, corners of squares, doorways in which a person may be concealed and suddenly rush out to commit the crime.

Par ses comparaisons avec des contrées éloignées, littéralement exotiques pour l'essentiel de son lectorat peu habitué à voyager, la presse entretenait également l'idée selon laquelle certains lieux étaient des creusets de marginalité absolue. Le Times par exemple déclarait en 1900 que certaines zones de Whitechapel étaient plus dangereuses pour le passant que les coins reculés de Calabre, de Sicile ou de Grèce ${ }^{49}$. 
29 Mais la presse n'était pas la seule à occuper l'univers du fantasme et de l'invention. S'agissant des bandes plus ou moins délinquantes de la capitale, le flou des formulations des observateurs de tous les horizons le disputait au ton péremptoire de leurs jugements de valeur : on parlait de 'small mob of boys', de 'idle ruffians', etc. ${ }^{50}$

Le monde de la prostitution subissait les mêmes distorsions : on parlait de 'harpies' ou de 'swarms of fallen women ${ }^{51}$. Les enfants n'échappaient pas à la règle. On les évoquait en des termes propres à décrire les animaux sauvages: 'wild', 'swarming', 'prowling', 'roaming'52. Les guides touristiques, notamment étrangers, n'étaient pas en reste, comme l'indique cet avertissement figurant au tout début de la dixième édition (1900) du célèbre guide Baedeker pour Londres :

Nous recommanderons du reste à l'étranger d'être continuellement sur ses gardes et surtout de faire toujours attention à sa bourse et à sa montre, car Londres fourmille de voleurs et d'escrocs d'une adresse inouïe, auxquels l'habitant de Londres lui-même n'échappe qu'avec peine. On devra particulièrement se rappeler cette recommandation en montant (...) en omnibus, aussi bien qu'en descendant, en somme partout où il y aura foule. (...) Éviter aussi, le soir, les quartiers pauvres et les rues écartées ${ }^{53}$.

31 Plus que d'autres peut-être, le journaliste Henry Mayhew a fixé dans maints esprits les caractères fondamentaux d'une certaine "marginalité ", en choisissant d'intituler son œuvre London Labour and the London Poor. Chez lui, les « gens de la rue » ('street folk', c'està-dire prostituées, pickpockets, mendiants, marins, etc.) - qu'il perçoit comme étant plus ou moins en rupture de ban avec la société ${ }^{54}$ - sont souvent assimilés au groupe beaucoup plus vaste constitué par les pauvres en général ${ }^{55}$.

32 L'historienne Gertrude Himmelfarb, s'intéressant entre autres à Mayhew, a d'ailleurs montré que classes dominantes et observateurs de la société britannique (ceux-ci issus de celles-là) ont confondu les turpitudes d'une minorité de pauvres avec le quotidien parfaitement banal quant à lui - de tous ceux qui éprouvaient des difficultés économiques. En prenant comme dénominateur commun les cas de figure les pires, tous en sont arrivés à formuler des "solutions» totalement inadaptées pour l'écrasante majorité des pauvres :

Then, as now, what passed as 'the culture of poverty' was the culture of a small subgroup of the poor. Yet that culture, or more precisely the image of that culture, was of momentous consequence in shaping the lives not only of the poor but of society as a whole..$^{56}$

Comme, entre-temps, une partie de la population londonienne avait été marginalisée géographiquement, cela ne manquait pas de renforcer le pouvoir de ce discours. La construction, en effet, de gares et de voies ferrées, de nouvelles artères, de canaux, etc., responsables de la disparition de nombreux logements, avait entrâné de profonds bouleversements.

D'une part, la mobilité des pauvres s'accroissait d'autant. À la fin des années 1880, l'un des membres du bureau scolaire de Bethnal Green indiquait à Charles Booth que des 1204 familles répertoriées dans ses carnets (comprenant en tout 2720 enfants), 530 (soit 1450 enfants) avaient déménagé au cours d'une seule année. Elles n'allaient en règle générale jamais très loin, mais comme l'écrivait Booth, elles se déplaçaient tels « des poissons dans une rivière $" "^{57}$. Ce qui ne pouvait que conforter dans leur opinion ceux qui les percevaient déjà comme des groupes humains vivant en marge. D'autre part, ces milliers de personnes n'en finissaient pas de s'entasser en des lieux dès lors toujours plus exigus et isolés, au cœur même de la ville, car les réalisations mentionnées ci-dessus quadrillaient l'espace et 
faisaient naître bien des culs-de-sac où ne se rendaient plus guère que la police et les membres de missions caritatives. D'où cette déclaration d'Octavia Hill, devant le Committee on Artisans' Dwellings :

A great deal of the degradation of these courts is because no public opinion reaches them ; if you hear anybody talk about a cul de sac, and contrast it with any place that is a thoroughfare, you feel at once that it is the public opinion that affects the

character of a court more than police or anything else..$^{58}$

C'est tout naturellement qu'est né et que s'est imposé, loin de ces rues, le fameux concept des « deux nations $»^{59}$. Bien des Londoniens ont alors intériorisé ce réflexe qui consistait à ne fréquenter que certaines rues, les autres faisant partie d'un univers situé en dehors des circuits effectués quotidiennement parce qu'ils étaient tout simplement perçus comme des réceptacles de marginalité. Tout cela explique en outre pourquoi, pour lutter contre elles, on a eu recours à la police et à un certain discours moral, et permet de mieux comprendre la véritable nature des mesures prises par le législateur.

\section{Surveiller, réprimer, exclure, assimiler}

En s'appuyant essentiellement sur l'agent de police, les autorités londoniennes ont tout fait pour tenter de circonscrire ce/ceux qui pouvai(en)t échapper à leur regard. On quadrillait l'espace en ayant recours au fameux beat. Ainsi, le 29 avril 1833, Charles Rowan, l'un des deux responsables de la police métropolitaine, déclarait :

I think I may say safely, that every street, road, lane, court and alley within the Metropolitan Police District is visited constantly day and night by some of the police; the whole is divided into beats; those beats are all registered and may be referred to at any time. ${ }^{60}$

La police devait tout connaitre des rues: le nom des habitants, leurs occupations, etc., pour mieux distinguer les marginaux. Elle surveillait les maisons close $^{61}$; elle avait recours à des agents en civil dans Sclater Street (Brick Lane) et Whitecross Street lors des marchés dominicaux ${ }^{62}$. La nuit, dans les quartiers où l'éclairage public était défaillant (Whitechapel, Shadwell, Spitalfields, Stepney, etc.), le nombre d'agents avait tendance à être plus élevé6 ${ }^{6}$. C'est pourquoi la police en uniforme représentait pour beaucoup le seul rempart fiable contre toutes les formes d'inconnu:

The policeman is essentially necessary to the well being of the state; he it is who keeps in order the truculent and blood-thirsty mob, who, but for him, would at once rise upon their betters, deprive them of their lives and property, overthrow the Church, subvert the government, and dethrone the Queen. ${ }^{64}$

Néanmoins, les Victoriens ont aussi opposé leur «tout-moral » à ce qu'ils percevaient comme de la marginalité. Lorsque la plus importante des organisations caritatives l'Armée du Salut - allait, par l'intermédiaire de ce que l'on appelait The Social Wing of the Army, au devant des prostituées par exemple, dans la rue même, la véritable finalité de la démarche dépassait largement la simple volonté d'aider matériellement ces femmes: on leur parlait surtout de Dieu, des vertus du travail, de la tempérance, de l'amour de son prochain et de la fidélité conjugale.

De son côté, l'Open Air Mission, fondée en 1853, offrait certes emploi et logis aux garçons les plus pauvres, mais on exigeait en contrepartie qu'ils allassent par exemple à l'office le dimanche ${ }^{65}$. De la même manière, les divers arrondissements de la capitale qui tentaient tant bien que mal de venir en aide aux mendiants et vagabonds en les admettant dans 
leurs centres d'accueil, ne prodiguaient des soins et n'offraient de la nourriture qu'en échange d'une certaine discipline fondée sur le travail et la tempérance ${ }^{66}$.

Fait très révélateur, le rapport de la Charity Organisation Society pour l'année 1876 rappelait la nécessité de promouvoir le goût de l'effort personnel ('self-help'), de lutter contre un état d'esprit de dépendance "fatal à tout progrès " ${ }^{67}$. Comme le fait encore remarquer Gareth Stedman Jones dans son ouvrage capital pour comprendre les problèmes sociaux de Londres au XIX ${ }^{e}$ siècle, les passages de l'œuvre de Mayhew cités par cette organisation dans les années 1870, n'étaient jamais ceux qui analysaient les causes de la misère, mais ceux qui traitaient des stratagèmes subtils employés par les faux mendiants et vagabonds ${ }^{68}$. Il n'est pas surprenant non plus que, dans le Metropolitan Police Act adopté le 17 août 1839 afin d'améliorer les dispositions du Vagrancy Act de 1824, le chapitre 54 (section 13), réservé à la question de la prostitution, n'ait envisagé le sujet que du point de vue des passants et des riverains de telle ou telle rue, que l'on tentait par là de protéger d'un phénomène perçu par définition comme totalement étranger et blâmable. Avec le Contagious Diseases Act de 1866 tout d'abord, puis en raison des lois de 1868 et 1869 , la prostituée sera même classée sans ambiguïté parmi les pauvres considérés comme n'étant pas respectables, c'est-à-dire dans cette catégorie criminelle et semi-criminelle occupant le bas de la hiérarchie sociale établie en fin de siècle par Charles Booth. Tant par la fermeture au début des années 1870 de nombreux établissements plus ou moins bien fréquentés du West End (la zone de Haymarket notamment) que par la destruction de 200 lupanars dans l'East End en 1887 par exemple ${ }^{69}$, on repoussera du reste la prostituée vers la rue; bref, on la marginalisera pour de bon en l'obligeant à adopter un comportement social inhabituel où la rue remplacera finalement tous les éléments qui avaient peu ou prou structuré sa vie jusque là. Les dispositions de la loi de 1891 sur le vagabondage, qui renforceront celles de la loi de 1824, achèveront par ailleurs de marginaliser la prostituée; elles la classeront, ainsi que les homosexuels, parmi les mendiants et les vagabonds, tout en augmentant les peines encourues pour racolage ${ }^{70}$.

De la même manière, il s'agissait de faire payer sa noirceur morale au pauvre puisqu'elle seule, pensait-on, expliquait la délinquance dans son ensemble. Le battage fait par la presse concernant les vols avec violence commis dans les rues de Londres a induit, par le truchement de la réaction d'une partie de la population, une mutation sensible de la législation concernant la délinquance, marquée par une plus grande sévérité. Les violences perpétrées contre des passants au cours de l'année 1856 donnèrent naissance au Penal Servitude Act de 1857. Celles ayant eu lieu durant la seconde moitié de 1862 furent à l'origine de la loi votée en 1863 (Security Against Violence Act), communément appelée The Garotters' Act ${ }^{71}$. Le Penal Servitude Act (1864) et le Prisons Act (1865) tendirent en outre à rendre le séjour carcéral des délinquants plus dur. Le Habitual Criminals Act (1869) vint renforcer l'ensemble en imposant une période de contrôle policier de sept ans à tout criminel récidiviste ${ }^{72}$. Le Prisons Act de 1877 confirma cette orientation en consacrant la sévérité comme arme suprême.

43 Concernant les enfants errants, les années qui suivirent l'adoption de la loi Forster de 1870 virent l'instauration d'un système de surveillance qui visait à réduire à néant les zones d'ombre. Leurs repaires diurnes, qu'ils eussent été mendiants ou tout simplement oisifs, furent désormais surveillés par des philanthropes et des 'school-attendance officers', chargés de patrouiller les rues et les courées. Une fois repérés, ceux des enfants qui se trouvaient sans domicile fixe étaient envoyés dans des 'pauper schools', ou bien des 'industrial schools'. Ces contrôles s'intensifièrent après 1880, l'Education Act voté cette 
année-là autorisant des 'truancy officers' à poursuivre leurs enquêtes à l'intérieur des maisons. Après 1900, le système de scolarisation à mi-temps disparut pour arracher définitivement les enfants à la rue $\mathrm{r}^{73}$.

Le législateur a donc beaucoup travaillé et dans bien des domaines. A-t-il pour autant réglé la question de la véritable marginalité ? Point du tout, puisqu'il ne s'est pas attaqué - loin de là - à celle de la pauvreté. Il est vrai qu'à Londres, cela se serait avéré particulièrement difficile. Car, contrairement à ce qu'imaginaient bien des Londoniens, les hasards de la vie, l'intempérance, la fainéantise ou bien encore l'imprévoyance, conduisaient finalement peu à la rue. Dans la plupart des cas, on y finissait comme prostituée, désœuvré ou vagabond et mendiant en raison surtout de la nature de l'économie londonienne, avare d'emplois.

Ceux en effet que l'on disait marginaux, pourtant bien intégrés à une société dont ils essayaient, tant bien que mal, de tirer eux aussi avantage, ne faisaient malgré tout pas partie intégrante de l'ordre économique établi, Londres - plus que les autres villes du Royaume-Uni - ne possédant pas d'industries suffisamment développées pour donner des emplois permanents à l'ensemble de sa population ${ }^{74}$. Ces données objectives contribuaient sans aucun doute à façonner les mentalités :

London represented the problem of casual labour in its most acute form, and the fears engendered by the presence of a casual labouring class were naturally at their greatest in a city which was both the centre and the symbol of national and imperial power. Such fears permeated conservative, liberal, and socialist thought alike $e^{75}$.

Le thème de la marginalité dans les rues de Londres au XIX ${ }^{e}$ siècle est indissociable de la difficulté que la ville a eu à se structurer en raison de l'afflux massif de populations venues des quatre coins du pays et du monde. Bas salaires et emplois permanents en nombre insuffisant ont directement contribué à rejeter tout un ensemble de personnes aux marges de la société. Pourtant, d'exclus de la société, de marginalisés par elle, les moins bien lotis en sont venus à être considérés comme des personnes ayant choisi sciemment la vie à la marge, en raison, disait-on, de leurs carences morales. La prostituée s'impose ici comme une parfaite illustration de ce raccourci.

Ce qui était vrai de Londres l'était à n'en pas douter des autres villes du Royaume-Uni ; on retrouvait partout les mêmes réflexes fondés sur les mêmes préjugés. Interrogé par une commission de la Chambre des Communes en 1852 au sujet des jeunes délinquants, un certain Matthew Davenport Hill, juge à Birmingham, déclarait qu'il existait, selon lui, dans sa ville, plusieurs catégories de jeunes criminels. Toutefois, en y regardant de plus près, on s'aperçoit que chacune d'entre elles correspondait à un type de marginalité défini exclusivement suivant des critères d'ordre moral et social en vogue à l'époque : enfants de délinquants, enfants illégitimes, orphelins, enfants trouvés ou adoptés ${ }^{76}$. Par ailleurs, et très logiquement, Hill niait que la pauvreté pût être la cause première de la délinquance juvénile ; c'était essentiellement l'« indigence morale » de ces jeunes gens qu'il fallait incriminer, opinion que la commission semble avoir acceptée sans s'interroger outre mesure. Certes, vers le tournant du siècle, ce discours sera - partout au RoyaumeUni - nettement en perte de vitesse, préfigurant les avancées sociales du début du $\mathrm{XX}^{\mathrm{e}}$ siècle, fondées sur une nouvelle approche du problème de la pauvreté, plus réaliste, car moins moralisatrice. 


\section{NOTES}

1. Jacques LEMAIRE, "Marge, marginalité, marginalisme ", p. 7 in Jacques LEMAIRE (dir.), Les Marginalismes, Eds. de l'Université de Bruxelles (La Pensée et les hommes, $31^{\mathrm{e}}$ année, $\mathrm{n}^{\text {elle }}$ série, 9), 1988.

2. Daniel COENEN, «Aspects de la marginalité occidentale aux $X^{e}$-XVI ${ }^{e}$ siècles ", p. 39 in LEMAIRE, op.cit.

3. Yves BAREL, La Marginalité sociale, Paris : PUF, 1982, cité par Martine XIBERRAS, Les Théories de l'exclusion : Pour une construction de l'imaginaire de la déviance, Paris : A. Colin / Masson, 1996, p. 123.

4. Voir Flora TRISTAN, Promenades dans Londres, ou l'aristocratie et les prolétaires anglais [1842], Paris: Maspéro, 1983, pp. 132-133, note 11.

5. Voir par exemple : The Parochial Critic, $\mathrm{N}^{\circ} 78,24$ June 1868, p. 2 ; 'Letter to the Right Honourable Henry A. Bruce, Her Majesty's Principal Secretary of State for the Home Department', in Public Record Office / Home Office Papers (= PRO/HO ci-après) 45/9511/17216/16 et réponse du 17 mars 1870, in PRO/HO 45/9511/17216/17 ; Lettre de G.W. MURNANE, in PRO/HO 45/9511/17216/39 ; etc.

6. Charles ВООТН, Life and Labour of the People in London, First Series - Poverty, Vol. I - East, Central and South London, [1889-1902] New-York: Augustus M. Kelley Reprints, 1969, p. 186, et $3^{\text {rd }}$ Series Religious Influences, Vol. III - The City of London and the West End, London: Macmillan \& Co., 1902, p. 87.

7. Robert D. STORCH, 'Police Control of Street Prostitution in Victorian London : a Study in the Contexts of Police Action' in David H. BAILEY (ed.), Police and Society, Beverly Hills: Sage Publications, 1977, p. 66.

8. John TIMBS, Curiosities of London, [1855] London: Virtue \& Co. Ltd., 1877 ?, p. 42.

9. George Augustus SALA, Gaslight and Daylight, with Some London Scenes they Shine upon, London: Houlston \& Wright, 1859 ?, p. 1, et Françoise BARRET-DUCROCQ, Pauvreté, charité et morale à Londres au XIX ${ }^{e}$ siècle : Une sainte violence, Paris: P.U.F, 1991, p. 100.

10. Henry MAYHEW, London Labour and the London Poor [1861-1862] vol. iii, New York: Dover Publications Inc., 1968, p. 407.

11. Max O'RELL (pseud. de Paul BLOUET, professeur, ancien officier de cavalerie, qui se fixa en Angleterre en 1873), John Bull et son île: Mœurs anglaises contemporaines, [1869 ?] Paris : CalmannLévy, $1883,5^{\mathrm{e}}$ éd., p. 81.

12. Sir Charles REED, 'Elementary Education', Night and Day (mensuel publié par le docteur Barnardo), $\mathrm{N}^{\circ} 1,15$ January 1877, p. 3.

13. William J. FISHMAN, The Streets of East London, London: Duckworth, 1979, p. 126.

14. Cf. document mis en annexe de T.J. BARNARDO, Saved from Crime : Incidents in the Life of a Waif and Stray, London: Shaw \& Co., 1888 ?

15. Henry MAYHEW, op.cit., vol. IV, p. 277.

16. Voir pp. 280-281.

17. Voir pp. 282-283. C'était la spécialité des mutchers. Cf. William J. FISHMAN, The Streets of East London, p. 105.

18. Rob SINDALL, Street Violence in the Nineteenth Century : Media Panic or Real Danger?, Leicester: Leicester University Press, 1990, pp. 10-11.

19. George GODWIN, Another Blow for Life, London: W. H. Allen \& Co., 1864, p. 73.

20. William J. FISHMAN, East End 1888 : A Year in a London Borough among the labouring Poor, London: Duckworth, 1988, p. 192. 
21. Voir p. 183, et Charles BOOTH, op.cit., $3^{\text {rd }}$ Series, Religious Influences, Vol. II, London North of the Thames: The Inner Ring, London: Macmillan \& Co., 1902, p. 245.

22. William J. FISHMAN, East End 1888, pp. 182-183.

23. Henry MAYHEW, op.cit., vol. III, p. 393.

24. 'Labour and the Poor - Part 1', Tracts by Christian Socialists, III, (reprinted with a few alterations from Fraser's Magazine for January 1850), London: J.J. Bezer, s.d., pp. 8-9. Voir également Henry MAYHEW, 'Letter VIII', Morning Chronicle, 13 November 1849, pp. 146 et suivantes in E.P. THOMPSON et Eileen YEO (eds.), The Unknown Mayhew : Selections from the Morning Chronicle 1849/1850, London: Merlin Press, 1971 ; Hippolyte TAINE, Notes sur l'Angleterre, Paris : Librairie Hachette \& Cie., 1876, $5^{\text {e }}$ éd., p. 39 ; et Jack LONDON, The People of the Abyss [1903] London: The Journeyman Press, 1992, pp. 114-115.

25. Voir E. Royston PIKE, Human Documents of the Victorian Golden Age (1850-1875), London: George Allen \& Unwin Ltd., 1967, p. 345.

26. Judith R. WALKOWITZ, 'Jack the Ripper and the Myth of Male Violence', Feminist Studies, Vol. 8, N³, autumn 1982, p. 554. Cf. L.C.B. Seaman, Victorian England: Aspects of English and Imperial History: 1837-1901, [1973] London: Routledge, 1990, p. 305.

27. Voir E. Royston PIKE, op.cit., p. 350.

28. Henry MAYHEW, op.cit., vol. III, pp. 369 et 375.

29. Henry MAYHEW, op.cit. vol. I, p. 105.

30. James WINTER, London's Teeming Streets (1830-1914), London: Routledge, 1993, p. 138.

31. Martine XIBERRAS, Les Théories de l'exclusion, p. 126.

32. Henry MAYHEW, op.cit., vol. IV, pp. 315 et 330-331. Voir pp. 300 et suivantes pour un exposé complet de la question.

33. Émile de LA BÉDOLLIÈRE, Londres et les Anglais, Paris : Gustave Barba, 1862, p. 309.

34. Godfrey Holden PIKE, Beneath the Blue Sky: Preaching in the Open-Air, London: Hodder \& Stoughton, 1888, p. 18.

35. John STEVENSON, Popular Disturbances in England,1700/1870, London: Longman, 1979, p. 163.

36. Alan PALMER, The East End : Four Centuries of London Life, London: John Murray, 1989, p. 66. Pour un exposé complet, voir l'article du Morning Star (18 January 1861), in John HOLLINGSHEAD, Ragged London in 1861 [1861] intr. A. S. WOHL, London: Dent, 1986, p. 5. Cf. Gareth STEDMAN JONES , Outcast London: A Study in the Relationship between Classes in Victorian London, Oxford: The Clarendon Press, 1971, pp. 45-46.

37. Roland MARX, La Reine Victoria, Paris : Fayard, 2000, p. 321.

38. Gareth STEDMAN JONES, op.cit.,, pp. 292-294.

39. Voir à ce sujet Arlette FARGE, La Vie fragile: Violence, pouvoirs et solidarité à Paris au XVIII siècle, Paris : Hachette, 1986, p. 198.

40. D'après A. L. Beier, à l'époque déjà, un tiers seulement des vagabonds erraient pour mendier et voler; on trouvait parmi les autres des maçons, des boulangers, des domestiques, etc., tous sans exception à la recherche d'un emploi, première cause de l'errance et de la mendicité inhérente à leur mode de vie très précaire. Voir Bronislaw GEREMEK, Inutiles au Monde : Truands et misérables dans l'Europe moderne (1350-1600), Paris : Gallimard/ Julliard (Coll. Archives), 1980, pp. 71-72 et 96-106.

41. Michel FOUCAULT, Surveiller et punir : Naissance de la prison [1975] Paris : Gallimard, 1993, pp. 90-91.

42. Voir notamment Robert D. STORCH, 'The Policeman as Domestic Missionary: Urban Discipline and Popular Culture in Northern England, 1850-80' in R.J. MORRIS \& Richard RODGER (eds.), The Victorian City : A Reader in British Urban History (1820-1914), London: Longman, 1993, pp. 304-305.

43. Daniel COENEN, " Aspects de la marginalité occidentale aux $\mathrm{XV}^{\mathrm{e}}-\mathrm{XVI}{ }^{\mathrm{e}}$ siècles ", in LEMAIRE, Les Marginalismes, p. 46

44. Martine XIBERRAS, Les Théories de l'exclusion, p. 119. 
45. Cf. Jennifer DAVIS, 'The London Garotting Panic of 1862' in V.A.C. GATRELL, Bruce LENMAN \& Geoffrey PARKER (eds.), Crime and the Law : The Social History of Crime in Western Europe since 1500, London: Europa Publications Ltd., 1980, p. 204.

46. Geoffrey PEARSON, Hooligan : A History of Respectable Fears, London: Macmillan Education Ltd., 1987, pp. 130-131.

47. Cité par Rob SINDALL, Street Violence in the Nineteenth Century, p. 54.

48. Ibid. pp. 39 et 40.

49. The Times, 30 October 1900, cité par Geoffrey PEARSON, op.cit.,, p. 76.

50. ALPHA (pseud.), The Outrages at St. George's in the East : A Letter to Sir George Cornewall Lewis, London: Joseph Masters, 1860, p. 4.

51. The East London Observer, 23 janvier 1858, cité par Chaim BERMANT, Point of Arrival: A Study of London's East End, London: Eyre Methuen, 1975, p. 185.

52. Anna DAVIN, Growing up poor : Home, School and Street in London, 1870-1914, London: Rivers Oram Press, 1996, p. 15.

53. Karl BAEDEKER, Londres et ses environs, Leipzig : K. Baedeker, $1900\left(10^{\mathrm{e}}\right.$ éd. refondue et mise à jour), pp. 1-2.

54. Henry MAYHEW, op.cit., vol. I, pp. 2-3.

55. Gertrude HIMMELFARB, 'The Culture of Poverty', p. 712 in Harold J. DYOS \& Michael WOLFF (eds.), The Victorian City : Images and Realities, Vol. II, London: Routledge \& Kegan Paul, 1973. Pour une critique de fond de l'œuvre de Mayhew, voir également A.S. WOHL, 'Introduction', p. xvii in John HOLLINGSHEAD, Ragged London in 1861.

56. Gertrude HIMMELFARB, 'The Culture of Poverty', p. 731 in Harold J. DYOS \& Michael WOLFF (eds.), The Victorian City, Vol. II.

57. Charles BOOTH, op.cit., First Series, Vol. I, pp. 26-27.

58. Cité par Gareth STEDMAN JONES, Outcast London, p. 180.

59. Voir David CANNADINE, 'Residential Differentiation in Nineteenth-Century Towns : from Shapes on the Ground to Shapes in Society' in James H. JOHNSON \& Colin G. POOLEY (eds.), The Structure of Nineteenth Century Cities, London: Croom Helm, 1982, pp. 239 et 245.

60. 'Select Committee on Metropolitan Police', in Parliamentary Papers 1834, Vol. XVI, folio 27.

61. Robert D. STORCH, 'Police Control of Street Prostitution in Victorian London' in David H. BAILEY (ed.), Police and Society, p. 68, note 5.

62. Godfrey Holden PIKE, Beneath the Blue Sky, pp. 7 et 21.

63. George R. SIMS, London by Night, London: Greening \& Co., 1910, p. 76.

64. Paul PRENDERGAST, 'The Policeman' in Heads of the People: or, Portraits of the English, [1840] Vol. II (dessins de Kenny MEADOWS, et textes de Douglas JERROLD, W.M. THACKERAY, Leigh HUNT, Mrs. S.C. HALL et al.), London: Henry G. Bohn, 1864, pp. 250-251.

65. James WINTER, 'The Agitator of the Metroplis : Charles Cochrane and Early Victorian Street Reform', London Journal, Vol. 14, $\mathrm{N}^{\circ} 1,1989$, p. 40. Voir aussi The Shoe-Black Societies and the Street Shoe-Blacks, 1860 ?, p. 1, in PRO/HO 45/OS. 7205, et 'The Independent Shoe-Black', p. 133 in John THOMSON \& Adolphe SMITH, Victorian London Street Life in Historic Photographs [titre original : Street Life in London], New York : Dover Publications, Inc., 1994.

66. Charles BOOTH, Life and Labour of the People in London, First Series, Vol. I, p. 221.

67. Voir Roland MARX, La Reine Victoria, p. 317.

68. Gareth STEDMAN JONES, Outcast London, pp. 10-11, surtout note 33.

69. Voir Judith R. WALKOWITZ, City of Dreadful Delight : Narratives of Sexual Danger in Late-Victorian London, The University of Chicago Press, 1992, p. 213, et Robert D. STORCH, 'Police Control of Street Prostitution in Victorian London', pp. 57 et 59 in David H. BAILEY (ed.), Police and Society.

70. Françoise BARRET-DUCROCQ, Pauvreté, charité et morale à Londres au XIXe siècle, p. 91. 
71. Ce type de délit devint alors le seul, en dehors d'actes de violence contre la reine, à pouvoir être puni du fouet. Cf. Jennifer DAVIS, 'The London Garotting Panic of 1862' in V.A.C. GATRELL, Bruce LENMAN \& Geoffrey PARKER (eds.), Crime and the Law, p. 208.

72. Voir p. 210.

73. Anna DAVIN, Growing up Poor, pp. 98-99.

74. Le point que nous touchons là est capital. En raison de la physionomie très particulière de l'économie londonienne, les réservoirs d'emplois stables pour les quantités innombrables d'ouvriers sans qualifications de la capitale étaient à vrai dire peu nombreux : 'Victorian London as a manufacturing centre concentrated as it had for centuries more on the provision of luxuries than of necessities, on consumer rather than on capital goods: silks rather than woollens; watches, pianos, jewellery, fine furniture, beer, spirits, sugar, tobacco rather than steel rails. Even more important to its economy were the service industries: hairdressing, education, tailoring, music, drama, domestic service, prostitution.' (Donald J. OLSEN, The Growth of Victorian London, London: B. T. Batsford, 1976, p. 325) On trouvait en revanche pléthore d'occupations temporaires (porteurs, serveurs, bouquetières), qu'offraient marchés, hôtels, clubs, boutiques ou théâtres, à tel ou tel moment de l'année plus qu'à tel autre. Voir Charles Booth, op.cit., First Series, Vol. I, pp. 227-228 ; Gareth STEDMAN JONES , Outcast London, p. 67 ; et Anthony SUTCLIFFE, 'In Search of the Urban Variable: Britain in the Later Nineteenth Century' in Derek FRASER \& Anthony SUTCLIFFE (eds.), The Pursuit of Urban History, London: Edward Arnold, 1983, p. 248.

75. Gareth STEDMAN JONES, Outcast London, p. 1.

76. 'Report of the Select Committee on Criminal and Destitute Juveniles' (questions 389-394), Parliamentary Papers 1852, Vol. VII, in E. Royston PIKE ed., Human Documents of the Victorian Golden Age (1850-1875), pp. 146-147.

\section{RÉSUMÉS}

$\mathrm{Au} \mathrm{XIX}^{\mathrm{e}}$ siècle, les rues de Londres furent perçues - à tort - comme un véritable creuset de marginalité par une presse et des élites promptes à l'amalgame. Cet énorme contresens qui consistait à croire que se mêlaient sur la voie publique voleurs, mendiants, prostituées et autres, s'est accompagné d'une offensive tendant à rendre le pauvre moralement meilleur par tous les moyens. Car le pauvre ne pouvait être pauvre qu'en raison de sa malhonnêteté, de sa fainéantise, etc. Ce faisant, le débat sur la question de la pauvreté - grande responsable de cette marginalité perçue - n'a pas vraiment eu lieu.

Both the capital's better-off classes and the press, who were always quick to denounce what they saw as the unacceptable ways of the poor, tended, mistakenly, to see the streets of nineteenthcentury London as places essentially characterised by prostitution, begging and thieving. Indeed, this misinterpretation resulted in an attack on the poor, aimed at raising raise them up morally, as their difficulties, it was thought, could only stem from improvidence, intemperance, and, more generally, from a disposition to dishonesty. But fighting poverty as such - poverty which was the main reason why thousands of people could exist nowhere but on the fringe - never really featured high on the social and political agenda of the day. 


\section{AUTEUR}

\section{DIDIER REVEST}

Université de Nice-Sophia-Antipolis 